\title{
RIFLESSIONI SUL TESTO DELLA COMMEDIA DANTESCA
}

\author{
Nota del s.c. EMILIO PASQUINI (*)
}

(Adunanza del 27 novembre 2014)

SuNTO. - Dopo una breve carrellata sull'edizione nazionale delle Opere di Dante e sulla bibliografia essenziale intorno al testo del poema, E. P. punta l'obiettivo sulla svolta semplificatrice segnata dall'edizione Petrocchi della "antica vulgata" e sui posteriori tentativi "bédieriani" esperiti nelle edizioni Lanza e Sanguineti, col successivo avvio del coraggioso cantiere "lachmanniano" gestito da Paolo Trovato. Un complesso di ricerche integrabile con la proposta (sulla linea Padoan-Pasquini-Veglia) di una diffusione della Commedia per grappoli di canti, non senza interferenze dovute, fin dall'origine, a una trasmissione orale. Il saggio si conclude con una campionatura di apparenti lezioni adiafore (tratte dall'apparato Petrocchi), le quali si risolvono tutte con la riduzione sembra - ineccepibile di una delle due alternative ad iniziative copistiche che escludono ogni sospetto di varianti d'autore.

$* * *$

ABSTRACT. - After a brief overview of the national edition of Dante's works and the essential critical bibliography concerning the poem's text, the author focuses on Petrocchi's edition of the 'antica vulgata,' on the subsequent attempts by Lanza and Sanguineti (based on Bédier's method), and the generous experiment by Paolo Trovato (based on Lachmann's method). These proposals can be integrated with the idea (supported by Padoan, Pasquini, and Veglia) of a diffusion by groups of cantos characterised, from the outset, by the interference of oral transmission. The article also examines a number of supposedly indifferent variants (taken from Petrocchi's critical apparatus), where one of the two alternatives can be recognised as the copyist's initiative, so as to exclude any doubt that it provides an authorial variant.

(*) Università degli Studi di Bologna, Italia. E-mail: emilio.pasquini@unibo.it 
Mi piace esordire con un rapido accenno alla recente edizione delle Opere di Dante ${ }^{1}$, ancora "provvisoria", anche se in gran parte accoglie testi che figurano allestiti per l'edizione nazionale: in altre parole, $\mathrm{i}$ più autorevoli allo stato attuale. Nella prefazione, dovuta allo stesso Domenico De Robertis, si passano in rassegna i problemi specifici di ogni scritto dantesco, a partire dalla Vita nova per la quale si adotta l'edizione Gorni, rinunciando a quella barbiana, ma sopprimendone gran parte dei grafemi latineggianti: da ricondurre ai copisti e non certo all'ignoto usus scribendi dell'autore (di cui non possediamo autografo neppure un rigo). Quanto alla Commedia, non si poteva non rispecchiare l'edizione della "antica vulgata" dovuta alle cure di Giorgio Petrocchi (1966-67), pur nella consapevolezza che anche per il poema, come per le opere "minori", si sono via via aperti nuovi cantieri.

Non intendo certo ripetervi quanto facilmente potreste attingere ai contributi specifici per la storia del testo della Commedia, cominciando dalle 40 pagine che, in occasione del settimo centenario della nascita dell'Alighieri, Gianfranco Folena ha dedicato al poema entro l'ottantina riservata alla complessiva tradizione degli scritti di Dante': da integrare col paragrafo Tradizione del testo di Antonio Enzo Quaglio entro la voce Commedia stesa per l'Enciclopedia dantesca e col brillante ragguaglio dello stesso Quaglio Sulla cronologia e il testo della «Divina Commedia» (entro "Cultura e scuola" del 1965)3. Ma né Quaglio né tanto meno Folena (il quale di Petrocchi conosceva solo i saggi preparatorî, usciti fra il 1955 e il 1958) erano in grado di sottolineare quello che oggi appare a tutti chiaro: che, cioè, l'edizione Petrocchi avrebbe segnato per vari anni una pausa negli studi filologici intorno al capola-

1 Finalmente uscite, con un ritardo dovuto alle note vicissitudini della Società dantesca italiana, a cura di Domenico de Robertis e Giancarlo Breschi, Firenze, Edd. Polistampa, 2012, sul modello del Dante del 1921, diretto da Michele Barbi (ristampato nel 1960). Se ne veda la Prefazione, firmata nel 2010 dallo stesso De Robertis.

2 La tradizione delle opere dantesche, in "Atti del congresso internazionale di studi danteschi”, I (20-27 aprile 1965), Firenze, Sansoni, 1965, pp. 1-76.

3 Vi si ponevano in grande risalto tre momenti dell'avventura filologica intorno al poema: la rinuncia del Vandelli (1921) alle procedure lachmanniane; il primo stemma codicum dovuto al Casella (1924); e i grandi meriti della semplificazione ecdotica introdotta dall'edizione Petrocchi (La "Commedia" secondo l'antica vulgata, Milano, Mondadori, 1966), nel suo limitarsi alla quasi trentina di codici anteriori alla normalizzazione editoriale del Boccaccio (1355). 
voro dantesco ${ }^{4}$, ben più intensa di quella che caratterizzò il periodo successivo all'edizione barbiana del 1921. Oltre alla perfetta documentazione di quel poco che sappiamo intorno alla composizione e alla pubblicazione della Commedia e del molto che invece ci è noto sulla sequenza degli approcci filologici al testo del poema ${ }^{5}$, Folena aveva però il merito di sottolineare l'improbabilità dell'esistenza di un archetipo emilianoromagnolo, postulata ma non dimostrata dal Casella ${ }^{6}$; e viceversa la prevalenza di una trasmissione orizzontale (dunque di una contaminazione) del testo rispetto alla normale, verticale, non senza turbative di ordine mnemonico ${ }^{7}$, fin dai primi esemplari superstiti ${ }^{8}$. La rarità di lacune meccaniche e di interpolazioni, data «la serratissima struttura numerica del poema», non impedirebbe, secondo Folena, la fiducia nella via maestra della recensio totale, sia pure di fronte a proposte, come quella di Petrocchi, euristicamente valide, oltre che ragionevoli?.

E tuttavia nell'introduzione alla sua edizione ${ }^{10}$, appena un anno dopo, Petrocchi muoveva proprio da una sfiducia nella recensio totale degli oltre 600 manoscritti del poema ${ }^{11}$, una sfiducia che era già maturata in Vandelli, nella sua ricerca della variante "archetipica", per rinuncia a una classificazione completa dei testimoni. A parere di Petrocchi, i 27 manoscritti dell'antica vulgata sono idonei «a coprire tutta la tessitura delle varianti indifferenti», in un'opera, come la Commedia, «mal sospettabile di varianti d'autore, nostro malgrado, in nessun suo luogo o parte» ${ }^{12}$. A Petrocchi, che pur ricostruiva il suo

4 Per gli anni successivi si può ricorrere al ricco panorama offerto da Marco Veglia, Sul testo della "Commedia" (da Casella a Sanguineti), in "Studi e problemi di critica testuale”, 66 (2003), pp. 65-119.

5 A partire dal precursore settecentesco, Bartolomeo Perazzini: Witte (1862), Moore (1894), Barbi (1891 e 1897), Vandelli (1902 e 1921), Casella (1923-24), infine Petrocchi (1955 e 1957). Cfr. Folena, La tradizione..., pp. 67 ss.

6 Ivi, p. 42.

7 Ivi, p. 47.

8 Mart in primo luogo (del 1548, collazione sull'Aldina del 1515, dove si riflette l'editio variorum del pievano Forese, 1330), il Landiano, ma anche il più tardo Laurenziano S. Croce trascritto da Filippo Villani.

9 Folena, La tradizione..., pp. 47-48 e 60-61.

10 La "Commedia" secondo l'antica..., I, Introduzione, pp. 3 ss.

11 Che oggi (grazie agli studi della Boschi Rotiroti e di altri) sappiamo essere oltre 800.

12 Petrocchi, Introduzione..., pp. 14-15 e 113. 
testo sulla base dello stemma dell' «antica vulgata», stemma dinamico perché «graduato nel tempo» (è forse questa la sua maggiore novità), non sfuggiva il pregio dell'Urbinate 366 (Urb), «testimone preminente di $\beta[\ldots]$, manoscritto di rara resistenza all'errore e, ciò che più interessa, alla contaminazione»; e di contro il primato del Trivulziano 1080 (Triv) sul piano linguistico, per la sua schietta fiorentinità ${ }^{13}$. Non a caso, negli anni successivi, i fautori del codex optimus avrebbero fatto ricorso a questi due testimoni.

Fra le tante recensioni all'edizione Petrocchi spicca ancora oggi, per equilibrio, quella dovuta a un filologo romanzo come Elio Melli ${ }^{14}$, specie in virtù dello sviluppo che egli dava alla discussione sulle varianti adiafore; in ogni caso, il consenso fu unanime e tale da imporre una sorta di pausa trentennale agli studi sul testo della Commedia. Fu quasi fatale che a quel punto, dopo tanto piétiner sur place, si imponesse l'opzione bédieriana per il bon manuscrit: si ebbe così, nel 1995, sul fondamento di Triv, l'edizione a cura di Antonio Lanza e nel 2001, sul fondamento di Urb, quella a cura di Federico Sanguineti. Quest'ultimo tuttavia, a differenza di Lanza, procedeva ad una verifica per campioni dell'intera tradizione del poema, giungendo a uno stemma bipartito dove $\beta$ è rappresentato dal solo Urb. Una scelta, quella di procedere per campioni, che non poteva andare esente da critiche, nel suo rimettere in campo la proposta di Barbi (accolta dalla Società dantesca italiana); così come a critiche radicali si prestava la sua scelta bédieriana, per un manoscritto di area emiliano-romagnola, necessariamente da "tradurre" in toscano. Si vedano ${ }^{15}$ le recensioni di Giorgio Inglese, ma soprattutto quella di Cesare Segre ( $«$ E' sicuro che molte lezioni dell'Urbinate siano semplicemente innovazioni del copista»); interessante anche l'intervento di Enrico Fenzi ${ }^{16}$ sulla celebre terzina di Purg. XXIV 55-57, atto di nascita della "scuola" del Dolce stil novo, dove Sanguineti legge «di qua dal dolce stil! e il novo ch'io odo»: la proposta

13 Ivi, pp. 368 ss., 404 ss., 413. Il copista, verso il 1337, fu Francesco di ser Nardo da Barberino.

14 Uscita nel "Giornale storico della letteratura italiana”, CXLVII (1970), pp. $111-132$

15 Rispettivamente in "La Cultura", XL (2002), pp. 483-508 e in "Strumenti critici”, 2 (2002), pp. 423-424.

16 In "Studi danteschi”, LXVIII (2003), pp. 67-82. 
finale di Fenzi suona per l'intera terzina «O frate, issa vegg'io - diss'egli - il nodo / che '1 Notaro e Guittone e me ritenne. / Di qua dal dolce stil è 'l novo ch'i' odo!».

Non meno significativi, a mio avviso, gli stimoli che vengono dal corposo intervento (2003) di Marco Veglia ${ }^{17}$, specie se lo si faccia interagire col successivo mega-cantiere aperto dall'intrepido Paolo Trovato, attraverso una serie di lavori preparator $\hat{1}^{18}$, anche di carattere metodologico ${ }^{19}$, approdati infine al magno volume Nuove prospettive sulla tradizione della «Commedia». Una guida filologico-linguistica al poema dantesco ${ }^{20}$, che non resta però l'approdo finale delle sue ricerche. Esse infatti sono proseguite nel 2009 con le Postille a una postilla. Il sub archetipo $\beta$ della «Commedia», $i$ luoghi barbiani e la contaminazione extra-stemmatica e con i Primi appunti sulla veste linguistica della «Commedia» ${ }^{21}$ : pagine fortemente polemiche nei confronti dell'edizione Lanza, sia per il primato accordato al Trivulziano (cui si contrappongono i toscanismi salvati dall'Urbinate), sia per la «sistematica discussione del troppo e del vano, ossia di varianti tipicamente adiafore ${ }^{22}$. Resta ferma, in Trovato, la persuasione che l'edizione "definitiva" della Commedia, al termine del suo lavoro di équipe non sarà molto diversa da Urb e dunque dall'edizione 2001 di Sanguineti. (Ma allora, mi domando, a che pro tanti sforzi? $)^{23}$.

Non voglio certo aggiungere una nuova recensione al corpus di

17 Già cit. alla nota 4 supra.

18 Per il testo della "Commedia". Varianti poziori di tradizione, in Studi in onore di P.V. Mengaldo per i suoi settant'anni, Firenze, Sismel, 2007, pp. 263-278; Postille sulla tradizione della "Commedia", in "Filologia italiana", IV (2007), pp. 73-77.

19 Sugli stemmi bipartiti. Decimazione, asimmetria e calcolo delle probabilità (in collaborazione con V. Guidi), in "Filologia italiana", I (2004), pp. 9-48; Archetipo, stemma codicum e albero reale, ivi ma II (2005), pp. 9-18.

20 Firenze, Franco Cesati, 2007.

21 Rispettivamente in "Studi danteschi", LXXIV (2009), pp. 307-315 e in "Medioevo romanzo", XXXIII (2009), pp. 29-48.

22 Primi appunti.., passim.

23 A tutt'altre motivazioni si ispira, credo, l'iniziativa di Art Codex e della Biblioteca Apostolica Vaticana per offrire un'edizione facsimile dell'Urbinate 365, alla quale mi hanno chiesto di collaborare con un'introduzione generale su Dante (doveva uscire entro il 2015, rinnovando analoga impresa di 50 anni fa, per il settimo centenario della nascita). 
Trovato, rispetto alle tante apparse fra il 2007 e il $2009^{24}$; ma piuttosto percorrere rapidamente i tratti essenziali del nuovo cantiere apertosi sotto il cielo di Ferrara sulla scorta del volume del 2007, che si apre con un generoso riconoscimento dei meriti di Petrocchi ${ }^{25}$, quasi smentito però da un successivo giudizio sulla «modesta attrezzatura filologica» dello stesso, specie in presenza dell'edizione Sanguineti, la quale riconduce la tradizione a un archetipo (escluso da Petrocchi) e ad uno stemma lachmannianamente produttivo ${ }^{26}$. Il principale risultato della semplificazione dello stemma (con Urb unico rappresentante di $\beta$ ) è che la Commedia del fiorentino Dante si sarebbe diffusa in tutta Italia attraverso un archetipo settentrionale ${ }^{27}$.

E tuttavia restano di qualche suggestione non tanto le osservazioni su Fuori dall'antica vulgata. Nuove prospettive nella tradizione della "Commedia"28, ove si specillano varianti di sostanza fin qui ignote o non tenute in considerazione, quanto l'accenno agli studi di Marco Veglia e di chi scrive, per l'originaria diffusione della Commedia attraverso gruppi o manipoli di canti ${ }^{29}$, anche alla luce dell'epistola di frate Ilaro ${ }^{30}$. Proprio questi accenni finali mi consentono qualche sviluppo, in un fecondo dialogo con certi spunti di Veglia: sia $^{31}$ sul problema della contaminazione (la intermixture di Moore o la mischianza di Barbi) e della

24 Particolarmente negativa quella di Enrico Malato (in "Rivista di studi danteschi", VII, 2, 2007, pp. 384-405) e soprattutto quella di Massimo Seriacopi (in "Rassegna della letteratura italiana", s. IX, 112, 2008, pp. 190-193), vera e propria stroncatura (a detta dello stesso Trovato, in un biglietto al sottoscritto, «feroce, ma senza motivazioni scientifiche»).

25 I cui risultati «ancor oggi tengono» (Nuove prospettive..., p. 9), anche dopo l'emergere di nuovi testimoni della antica vulgata: dai 27 di Petrocchi siamo passati agli 85 della Boschi Rotiroti (ivi, pp. 49 ss.).

26 Ivi, pp. 8 ss. e 12. Trovato però dimentica che dietro le scelte di Giorgio Petrocchi vigilava, con assiduo consiglio e controllo, nientemeno che Gianfranco Contini, allora Presidente della Società dantesca italiana.

27 Ivi, p. 229.

28 Ivi, pp. 669 ss.

29 Ivi, pp. 628 ss.

30 Dove però Trovato è fermo alle negazioni di Bellomo e ignora gli studi di Giuseppe Indizio. Cfr. almeno E. Pasquini, L'Epistola di frate Ilaro: un nuovo documento per l'autenticità, in "Lunigiana dantesca", Bollettino on-line del Centro lunigianese di studi danteschi, a. XII, n. 97 (luglio 2014), pp. 6-8.

31 Veglia, Sul testo..., pp. 67 ss. 
conseguente inapplicabilità di un rigoroso metodo lachmanniano ${ }^{32}$; sia sulla legittimità della linea Padoan-Pasquini ${ }^{33}$, del resto confortati dalle ricerche della Pomaro $^{34}$, e perfino da una lungimirante intuizione del Vandelli35: "è mera ipotesi che avesse pubblicato una cantica intera per volta $[\ldots]$ non c'è difficoltà a credere che la pubblicazione avvenisse anche per sezioni minori di una cantica [...] qui senza dubbio (nel racconto di Boccaccio) ci è attestata viva la tradizionale credenza in una pubblicazione spezzata e graduale, altrimenti fatta che con una cantica per volta". In altre parole, io non avrei dubbi circa la graduale diffusione di blocchi o grappoli di canti e circa l'impossibilità per l'autore di intervenire con correzioni su quanto già licenziato in pubblico ${ }^{36}$.

Di qui l'importanza dell'epistola di frate Ilaro (1314-15), a Inferno concluso e Purgatorio avviato alla conclusione ${ }^{37}$; di qui la possibilità che le copie integrali del poema, anche le più antiche, derivassero "da una costellazione di gruppi di canti in seguito assemblati" ${ }^{38}$. Tutto questo potrebbe spiegare sia l'eclissi di codici fra il 1321 e il 1331 (data di Mart, o meglio del perduto manoscritto di Forese Donati collazionato da Luca Martini a metà del '500), senza chiamare in causa $^{39}$ la "furia combustiva di Bertrando del Poggetto", non limitata certo alla Monarchia; sia la "verosimile inesistenza di un archetipo", sia l'improbabilità della "consueta bipartizione in $\alpha$ e $\beta$ " della tradizione

32 Quaglio, Tradizione del testo..., loc. cit.: "le testimonianze del poema sono ribelli ad ogni possibile reticolato genealogico".

33 G. Padoan, Il lungo cammino del "poema sacro. Studi danteschi, Firenze, Olschki, 1993, passim; E. Pasquini, Dante e le figure del vero. La fabbrica della "Commedia", Milano, B. Mondadori, 2001, specie pp. 1-26; Id., "Trasmutabile son per tutte guise" (Par. V 99): anticipazioni e compimenti nell'opera dantesca, in "Studi e problemi di critica testuale", 90 (aprile 2015), pp. 11-28.

34 Cit. da Veglia, Sul testo..., pp. 79-80.

35 Loc. cit.

36 Cfr. Veglia, Sul testo...., pp. 73 ss.; ma già Pasquini, Dante e le figure..., passim. Aggiungo in calce la mia persuasione che almeno alcuni canti non siano mai stati scritti dall'autore, ma da lui dettati a copisti per passione, amici o protettori, secondo una prassi millenaria, che va da Cicerone ai nostri umanisti del Quattrocento.

37 Veglia, Sul testo..., p. 80; Pasquini, Dante e le figure..., pp. 7-11.

38 Veglia, Sul testo..., , p. 81, il quale rinvia anche alla diagnosi di R. Abardo nella recensione all'edizione Sanguineti, in "Rivista di studi danteschi", I (2001), fasc. $1^{\circ}$, p. 156.

39 Col Livi cit. da Veglia, Sul testo...., p. 90. 
manoscritta ${ }^{40}$. Ricordiamo invece che Enrico Malato ${ }^{41}$ ammetteva la pubblicazione separata delle tre cantiche, con tre possibili ma improbabili archetipi; e che, pur confermando il suo fermo rifiuto di una diffusione per manipoli di canti, imbastiva una forte e meritoria difesa dell'edizione Petrocchi, rispetto alle tante riserve di Trovato, specie appellandosi alla forza invasiva della contaminazione.

In altre parole, anche a mio avviso, in attesa di altri possibili recuperi o estensioni dell'antica vulgata, meglio affidarsi alla schietta storicità dell'apparato critico dell'edizione Petrocchi ${ }^{42}$ : apparato su cui esercitare (in modo più maturo rispetto alla prassi artigianale del Vandelli) il iudicium del filologo, persuaso com'è dell'improbabilità di varianti d'autore anche di fronte ad apparenti adiaforie. E' quanto ho cercato io stesso di fare a più riprese $e^{43}$, proponendo alcuni esempi di scelte che, mirando al sistema interno (tematico e stilistico) dell'autore, vedessero prevalere una decisione univoca anche a dispetto di una rigida risposta dei codici. Mi limito qui a una parca ripresa di campioni, cominciando da Inf. XIV 46-48:

chi è è quel grande che non par che curi

lo 'ncendio e giace dispettoso e torto,

sì che la pioggia non par che '1 marturi?

maturi?

Sono le parole che Dante rivolge a Virgilio davanti alla figura gigantesca di Capaneo, fra i bestemmiatori del settimo cerchio, dove la tradizione si scinde fra il marturi di Landiano, Laurenziano XL.42, Riccardiano 1005 e Braidense AG.XII.2, rincalzato dal martiri di Ashburnhamiano 828, Madrileño 10186 e Fiorentino Palatino 313 (dunque manoscritti di $\alpha$ e di $\beta$ ), e il maturi di tutti gli altri testimoni della vul-

40 Ivi, p. 96.

41 Nella sua recensione (2007) al volume di Trovato più volte cit.

42 Contro la "metastoria" dell'edizione Sanguineti (cfr. Veglia, Sul testo..., p. 90). Un apparato che ci si augura possa integrarsi coi nuovi apporti per l'antica vulgata, in particolare gli 85 manoscritti primo-trecenteschi recuperati dalla Boschi Rotiroti.

43 Nella relazione Prolegomeni minimali al testo della "Commedia", con cui ho aperto l'anno accademico 2010-2011 nell'Accademia delle Scienze dell'Istituto di Bologna” (ora in "Rendiconti degli anni 2013-2014", Classe di Scienze morali, n. s., tomo VI, Bologna, Bononia University Press, 2015, pp. 75-84); ma insieme nel saggio Variazioni sul testo della "Commedia", in Una vita per la letteratura. A Mario Marti colleghi ed amici per $i$ suoi cento anni, a cura di M. Spedicato e M. Leone, Lecce, Edd. Grifo, 2014, pp. 319-320. 
gata. Ora, non vi è dubbio che quest'ultima sia l'unica lezione risalente all'autore, in quanto esiste un nesso inequivocabile fra questo verbo ${ }^{44} \mathrm{el}$ 'epiteto $a c e r b o^{45}$ riservato a quel Vanni Fucci (Inf. XXV 18), il quale è con Capaneo l'unico dannato cui Dante attribuisce le stimmate della superbia. Veniamo ora a Purg. XX 64 ss.:

Lì cominciò con forza e con menzogna

la sua rapina; e poscia, per ammenda, Pontì e Normandia prese e Guascogna.

Carlo venne in Italia, e per ammenda vittima fé di Curradino; e poi ripinse al ciel Tommaso, per ammenda...

Dove un bel manipolo di testimoni dell'antica vulgata, appartenenti sia al ramo $\alpha$ sia al ramo $\beta$ (Cortonese, Seminario di Belluno, Madrileño, Martini, Riccardiano 1010 e Trivulziano 1080),si oppone alla lezione tràdita dalla maggior parte dei codici antichi, con la triplicazione del sintagma per ammenda in rima, introducendo nel verso centrale la variante per vicenda, quanto mai idonea ad evitare la in apparenza fastidiosa triplicazione. Che si tratti però di un'innovazione copistica e non di variante d'autore, non ci sono dubbi: proprio alla luce di altre pregnanti triplicazioni in Dante: quella di Virgilio nel momento (Purg. XXX 49-51) in cui la guida scompare per lasciar posto a Beatrice, nella scia della triplicazione di Euridice nelle Georgiche virgiliane (dunque,

44 Come conferma anche l'esegesi (antica e moderna) più autorevole: da Benvenuto ("Videtur quod ignis fluens non mollificet duritiam eius") a Vellutello ("E' per similitudine de' frutti [...] Come 'l frutto è ben di pessima natura, quando per alcun tempo non si viene a maturare, così è l'ostinato..."), da Venturi ("metafora presa da' frutti, che deponendo l'acerbità e durezza, diventano maturi e mézzi") ad Andreoli ("che distrugge l'acerbità dell'animo suo, che lo domi"), con esito più consapevole in Tommaseo ("acerbi si dicono gli orgogliosi; acerbo è contrario di maturo, e la pioggia ammollisce le frutta cadendo"). Fuori strada, dunque, Gino Casagrande, che (in un saggio uscito sugli "Studi danteschi" del 2008) giunge a parificare acerbo a "bestia", epiteto già assunto su di sé dallo stesso Vanni Fucci (nell'auto-presentazione di Inf. XXIV 125-126).

45 Restano in superficie Benvenuto da Imola ("vocabat enim Vannem predictum, qui erat acerbus et crudus tamquam socium suum simillimum sibi"), Castelvetro ("Perché Caco cerca piuttosto Vanni Fucci che non altro? Come sa che egli sia più acerbo in Dio che gli altri, domandandolo per eccellenza 'l'acerbo'"?) e Andreoli ("l'autore di tante acerbe parole"); va a fondo invece e capisce quasi tutto Tommaseo: "Fucci, il duro, il mordace. Nell'Inf. XV chiama i Neri 'lazzi sorbi'; e di Capaneo: 'la pioggia non par che 'l maturi'”. 
per tre volte si invoca la persona cara che sta per scomparire definitivamente dalla nostra vita); oppure quella del sintagma il loco mio nella prima invettiva di san Pietro (Par. XXVII 22-23), ben giustificabile alla luce del fatto che il primo degli Apostoli è proprio l'uomo del tre: infatti per tre volte tradisce Cristo, negando di conoscerlo; per tre volte Gesù gli chiede "mi ami tu?" e per tre volte Pietro gli risponde affermativamente; infine per tre volte Cristo gli dice "Pasci il mio gregge".

Veniamo a un esempio riguardante la terza cantica, a Par. XXXI 20, un colpo d'obiettivo sopra l'aspetto della candida rosa, tra il volteggiare degli angeli e lo splendore delle vesti dei beati sui loro scanni:

Né l'interporsi tra 'l di sopra e 'l fiore

di tanta moltitudine volante

impediva la vista e lo splendore...

Così legge l'edizione nazionale curata da Petrocchi, del resto sul fondamento di uno schieramento (numericamente e qualitativamente) maggioritario dei manoscritti dell'antica vulgata ${ }^{46}$; ma un bel manipolo di testimoni reca al verso centrale la lezione plenitudine, che non può non apparire subito come la difficilior e per giunta quella più aderente al contesto. Si tratta di uno dei più geniali neologismi danteschi, coniato sul latino plenitudo, attestato in Columella e Plinio, ma soprattutto nella vulgata di san Girolamo e in Macrobio, autori ben familiari a Dante. Si riconferma così l'utilità dell'apparato critico dell'edizione Petrocchi, dalla cui escussione risulta palesemente l'assenza di varianti d'autore, già asserita dall'editore critico; unita alla persuasione che da una recensio completa della tradizione non possano venire mutamenti di qualche rilievo al testo del poema. Qualche miglioramento potrà invece essere indotto da una diversa interpunzione (settore nel quale i manoscritti antichi sono totalmente muti); e in questo senso chi vi parla ha già portato qualche contributo ${ }^{47}$.

46 Li elenchiamo: Ashburnhamiano 828, Cortonese, Napoli Girolamini, Ginori Venturi Lisci 46, Hamilton 203, Laurenziani XL.16 e XL.22, Madrileño, Martini, Riccardiano 1005 e Braidense AG.XII.2, Riccardiano 1010, Trivulziani 1080 e 1077, Urbinate 366.

47 Mi riferisco in particolare alla nuova lettura (avanzata già in Dante e le figure..., p. 277 e riproposta in vari interventi ancora inediti) di una terzina del canto di Ugolino (Inf. XXXIII 19-21), confermata dall'accertamento dell'inesistenza di altri cioè (congiunzione frequentissima nel prosatore) in tutto l'arco del poema; dunque: "Però quel che non puoi aver inteso, / ciò è come la morte mia fu cruda: / udirai e saprai s'ei m'ha offeso”. 